\title{
Color Dependence on Thickness in Topaz Crystal from Brazil
}

\author{
Waldemar Bonventi Jr, ${ }^{1}$ Sadao Isotani, ${ }^{2}$ and Antonio Roberto Pereira Albuquerque ${ }^{2}$ \\ ${ }^{1}$ Faculty of Technology of Sorocaba, Sorocaba, SP, Brazil \\ ${ }^{2}$ Institute of Physics, University of São Paulo, São Paulo, SP, Brazil
}

Correspondence should be addressed to Sadao Isotani, sisotani@if.usp.br

Received 22 March 2012; Revised 18 June 2012; Accepted 9 July 2012

Academic Editor: Mohindar S. Seehra

Copyright (C) 2012 Waldemar Bonventi Jr et al. This is an open access article distributed under the Creative Commons Attribution License, which permits unrestricted use, distribution, and reproduction in any medium, provided the original work is properly cited.

It is well known that crystals of topaz from the Eastern Brazilian Pegmatite Province may turn blue by the irradiation with ${ }^{60} \mathrm{Co}$ gamma rays followed by heat treatment. Also, it is known that the sensation of color changes with the thickness of these crystals. The dependence of the color, given by 1931 CIE chromaticity coordinates, with the thickness of the crystal was analyzed. The absorbance used in the calculation of these coordinates was given by the sum of Gaussian lines. The parameters of these lines were determined through the decomposition of the optical absorption spectra in the ultraviolet and visible regions. The decomposition revealed several lines, whose assignment was made considering studies in spodumene and beryl crystals and highly accurate quantum mechanical calculations. The transmittance becomes very narrow with increasing thickness, and the CIE chromaticity coordinates converge to the borderline of the CIE Chromaticity Diagram at the wavelength of maximum transmittance. Furthermore, the purity of color increases with increasing thickness, and the dominant wavelength reaches the wavelength of maximum transmittance.

\section{Introduction}

Most gemstone crystals have an intrinsic color due to the optical absorption and presence of color centers that are produced by impurities like transition metals and elements of Earth-rare, or defects in, the crystalline lattice [1]. However, variations in crystal color are produced by a combination of other factors, such as the form of the crystal and the light source [2]. The color shown by a gemstone reflects intrinsic factors like the actual form and presence of color centers as well as external factors like the light produced by different light sources.

The gemstone crystals used as jewelry, often undergo a process of cutting and polishing. The cutting and polishing of a crystal is a complex process. For this purpose, a crystal can be divided into several parts and with different shapes. In this process, it is emphasized the importance of getting these parts with color purity better defined. In this sense, the knowledge of the relation of color with the geometry may give subsidies for this purpose. Therefore, since the value of the gemstone is evaluated as a function of the exhibited color, a light effect prediction can be useful in the analysis of gemstones. The contribution of color centers, light source, and thickness can be evaluated using the $x y$ chromatic coordinates of the 1931 CIE (Comission Internationale d’Éclairage) system [3].

Gemological quality crystals of beryl, quartz, spodumene, topaz, and tourmaline among many others, constitute an important economic resource for the Eastern Brazilian Pegmatite Province [4]. The optical absorption spectra of crystals of the Province of Oriental Brazilian Pegmatite were reported by several authors for beryl [5-10], amethyst [11], spodumene [12-15], topaz [16-24], and tourmaline [25].

The topaz is one of the most mined gemstones in this region. The color of the topaz is caused by the presence of point defects induced by ionizing irradiation, which has been the subject of extensive study. The first study in early 1923 [16] described coloration from brown smoke to orange shades in topaz. In 1975, Nassau and Prescott reported a remarkable feature obtained from 86 colorless topaz samples with probable Brazilian origin; 21 samples exhibited a blue color after gamma irradiation followed by moderate heating [17]. This blue color in transmitted light is attributable to an optical absorption band near $620 \mathrm{~nm}$. 
The investigation of atomic models for point defects in topaz began to start only in 1990 when the $620 \mathrm{~nm}$ absorption band was attributed to an oxygen vacancy in an isolated $\mathrm{SiO}_{4}$ tetrahedron [19]. Later, a systematic study was reported on the effects of irradiation in topaz mined in different localities of Brazil, using electron paramagnetic resonance (EPR) and optical absorption [20-22]. It was shown that the absorption band at $620 \mathrm{~nm}$ induced by neutron or gamma radiation is responsible for blue color in topaz. Also, it was shown that a paramagnetic $\mathrm{O}^{-}$hole center is formed at a hydroxyl $\mathrm{OH}^{-}$. The $\mathrm{O}^{-}$hole center has the same thermal stability as the $620 \mathrm{~nm}$ absorption band. Further, Schirmer developed a model of $\mathrm{O}^{-}$hole centers in oxide materials, that is, a hole is trapped in a cluster of a few several equivalent oxygen ions next to an acceptor defect and is stabilized by lattice distortion as an $\mathrm{O}^{-}$bound small polaron [23]. Accordingly, Krambrock et al. discussed the $620 \mathrm{~nm}$ absorption band and $\mathrm{O}^{-}$hole centers in the context of $\mathrm{O}^{-}$ bound small polarons [24].

Crystals of topaz mined in the Eastern Brazilian Pegmatite Province may turn blue by irradiation with ${ }^{60} \mathrm{Co}$ gamma rays followed by heat treatment [18]. The blue colour is the result of the overlap of the absorption band at $620 \mathrm{~nm}$ and a tail of an absorption in the ultraviolet region. Also, it is known that the sensation of color changes with the thickness of these crystals.

We report in the present work the effect of thickness of topaz samples on the 1931 CIE $x y$ chromatic coordinates calculated using the absorbance described as the sum of Gaussian lines. The parameters of these lines were determined through a spectral decomposition analysis of the optical absorption in the visible and ultraviolet regions. The topaz samples were submitted to irradiation and heat treatment at $200^{\circ} \mathrm{C}$ and $600^{\circ} \mathrm{C}$. The parameters of these decompositions were refined using the optimization method reported by Isotani and Fujii [26]. Also, in this paper we report an analysis of the dependence of transmittance, color purity and dominant wavelength with thickness.

\section{Experimental}

The topaz samples used in the present work were obtained from the region around Governador Valadares, at the Eastern Brazilian Pegmatite Province, MG, Brazil and were classified as colorless topaz. Very small inclusions were distributed in the sample but did not change its transparency. The samples used in our study were oriented using the external morphology. The results of impurity analysis were reported in previous works $[18,27]$. The main impurity was of Fe (200-380 ppm), Cr (100 ppm), and Mn (60 ppm). Wafers of 10 to $50 \mathrm{~mm}$ thickness were cut from the samples using a diamond saw and polished using chromium oxide, alumina and diamond paste. The waffers were $\gamma$-irradiated using a ${ }^{60} \mathrm{Co}(\sim 400000 \mathrm{Ci})$ source from EMBRARAD SA at a rate of $80000 \mathrm{~Gy} / \mathrm{h}$. The dose was controlled by means of three processes: ceric-cerous dosimetric system, AECL red acrylic dosimetric system, and UKAEA red perspex dosimeter. Thermal treatments were made in air using a furnace, and the temperature was measured using a chromel-alumel thermocouple.

The optical absorption spectra used in the present work were recorded using a Carl-Zeiss DMR21 spectrometer.

\section{The 1931 CIE Chromaticity Diagram Calculations}

The human eye has receptors for three different wavelengths, that is, short (blue), middle (green), and long (red) wavelengths. Therefore, in principle, three coordinates describes the color sensation. The CIE XYZ is one of such spaces, based on measurements of human eye. Since the human fovea has an angular aperture $\theta$, the values of these coordinates are $\theta$ dependent. The $X Y Z$ coordinates of a light [1,2], coming from a light source denominated illuminant, that passes through a colored sample are given by

$$
\begin{aligned}
X_{\theta} & =\sum_{380}^{770} P(\lambda) \cdot T(\lambda) \cdot \bar{x}_{\theta, \lambda} \cdot \Delta \lambda, \\
Y_{\theta}= & \sum_{380}^{770} P(\lambda) \cdot T(\lambda) \cdot \bar{y}_{\theta, \lambda} \cdot \Delta \lambda, \\
Z_{\theta}= & \sum_{380}^{770} P(\lambda) \cdot T(\lambda) \cdot \bar{z}_{\theta, \lambda} \cdot \Delta \lambda,
\end{aligned}
$$

where $P(\lambda)$ is the spectral distribution of the illuminant, $T(\lambda)$ is the spectral transmittance of the sample, and $\bar{x}_{\theta, \lambda} ; \bar{y}_{\theta, \lambda} ; \bar{z}_{\theta, \lambda}$ are the color matching functions. In the present work, we use the illuminant A, a light source that represent a typical, domestic, tungsten-filament lighting and illuminant D65, a light source that represent the average daylight. The values of $\bar{x}_{\theta, \lambda} ; \bar{y}_{\theta, \lambda} ; \bar{z}_{\theta, \lambda}$ and $P(\lambda)$ can be obtained at CIE website [http://www.cie.co.at/index.php/Publications/Standards].

They were originally calculated through a complex algorithm using data obtained from experiments which correlate with the primary colors: red, green, and blue with the observed colors [3]. The angle $\theta$ in the present work was assumed to be the angle of maximum sensitivity $\theta=2^{\circ}$. Then, $\theta$ will be no more used in the following equations.

The 1931 Chromaticity Diagram is a projection of the CIE (Comission Internationale d'Éclairage) XYZ coordinate system in a two-dimensional plane $x y$ given by [3]:

$$
\begin{aligned}
& x=\frac{X}{X+Y+Z}, \\
& y=\frac{Y}{X+Y+Z} .
\end{aligned}
$$

The borderline of the 1931 Chromaticity Diagram is given by

$$
\begin{aligned}
& x_{\text {border }}=\frac{\bar{x}_{\lambda}}{\bar{x}_{\lambda}+\bar{y}_{\lambda}+\bar{z}_{\lambda}}, \\
& y_{\text {border }}=\frac{\bar{y}_{\lambda}}{\bar{x}_{\lambda}+\bar{y}_{\lambda}+\bar{z}_{\lambda}} .
\end{aligned}
$$


The open part of this diagram not covered by this transformation is closed by a straight line, called the purple line.

The calculation of color includes the effect of light absorption and illuminant. Therefore, a useful complement to the analysis of color is the analysis of color purity. The purity $p_{L}$ of a color is defined as the ratio of distances from the color and the dominant wavelength from a given illuminant and is given by

$$
p_{L}=\frac{x-x_{L}}{x_{\lambda}-x_{L}} \equiv \frac{y-y_{L}}{y_{\lambda}-y_{L}},
$$

where $\left(x_{L}, y_{L}\right)$ are the coordinates of the illuminant and $\left(x_{\lambda}, y_{\lambda}\right)$ are the coordinates of the dominant wavelength. The dominant wavelength of a color stimulus is the wavelength $\lambda$ that matches the given stimulus in color. It is evaluated as the cross-point of the diagram borderline and the line passing through the illuminant and the color coordinates.

When the line between color and light source coordinates reaches the purple line, the color does not possess a dominant wavelength. In this case, it is possible to match the specified achromatic stimulus with a mixture of the color and a monochromatic stimulus known as complementary wavelength $\lambda_{c}$. The complementary $\lambda_{c}$ is the point of the diagram borderline crossed by the straight line in the opposite direction from the light source to the color coordinates. The purity calculation will be similar to the dominant case, making use of (5). Since purity is a nondimensional number with a value between 0 and 1 , it is common to represent these values in percentages.

The crystal transmittance is given by

$$
T(\lambda)=T_{0} \exp (-\alpha(\lambda) \ell)
$$

where $\alpha(\lambda)$ is the absorbance and $\ell$ is the sample thickness. In the present case, the absorbance $\alpha(\lambda)$ can be expressed as the sum of absorption lines. Each line is expressed as a function of $\lambda$, and its form function is given by Gaussians, Lorentzians, pekarians, and so forth.

Each absorption line in the spectrum (as a function of the wave number $v=1 / \lambda$ ) has a Lorentzian shape in the absence of interaction between a defect and the crystal lattice. However, a crystal in nature is not perfect and has internal random stress. The absorption peak of individual atoms has a lorentzian shape, but a typical peak of some atoms absorbing nearly the same energy has a superposition of lorentzians. This "wrapper curve" can be approximated by several kinds of mathematical line shapes, like Gaussian, Lorentzian,Pekarian, or combinations of these. The interaction of phonons and defects may cause dependence between line shape and temperature. For ground and excited states potential energies with different minima, the shape results in a symmetrical curve at high temperature, which is usually satisfied at room temperature [28]. In addition, from approximate evaluation, the high temperature absorption should be Gaussian. Therefore, a Gaussian line shape was assumed for modeling the room temperature peak, and all spectra can be presented as a sum of these Gaussians:

$$
\alpha(v)=\sum_{i=1}^{n} a_{i} \exp \left[-\left(\frac{\nu-v_{0 i}}{\Delta v_{i}}\right)^{2} \ln 2\right]
$$

where $v$ is wave number, $\nu_{0 i}$ is the wave number of the $i$ th peak at its maximum, $\Delta v_{i}$, is the line width of the $i$ th peak, and $a_{i}$ is the amplitude of $i$ th component of the spectral line.

The method for determining the parameters of (6) by decomposition of the spectra of the topaz is a complex process because there are multiple overlapping peaks as well as peaks located outside the range of experimental measurement in the ultraviolet region. Moreover, weak ghost lines may arise due to poor propagation of errors in the fitting parameters. Finally, ghost lines can arise from weak non-Gaussian components introduced by any problem with the polarization detector nonlinearity and saturation, dispersing the sample surface defects due to polishing among other factors. So, we made spectral decomposition using as reference for the decomposition process applied prior to the absorption spectra of spodumene [15] and beryl [10].

The parameters of (6), $v_{0 i}, \Delta v_{i}$, and $a_{i}$, were determined using a grid optimization method [26]. This method was applied to optimize the decomposition of the spectra of spodumene [15] and beryl [10]. The procedure makes use of a grid in the parameter space obtained by changing each parameter back and forth and calculating robust cost functions on the surfaces of this grid. The lengths of the changes were determined empirically. The best set of parameters was calculated by the projection on the grid surface with the smallest cost function. This process was repeated until the cost function became smaller than a given value or after a previously chosen number of iterations. In the present study, we found that the decomposition is more efficient when the parameters of the Gaussians are determined using the grid method, and the amplitudes are calculated using the method of least squares.

In order to make the comparison between the results of decomposition of the different spectra, it is of fundamental importance to determine the deviations of the parameters. We have used the procedure developed for the determination of the deviations described by Isotani et al. [10]. Based on the error obtained in the best fit, we determined the deviation for an increase of the cost function from about $20 \%$. The highest and lowest values for each parameter were determined to correspond to $20 \%$ of the best fit of the cost function: the error in this parameter was assumed to be the biggest difference compared to that obtained in the best fit.

\section{Spectral Decomposition Analysis}

The chromatic coordinates described in (1) are dependent on the transmittance, which in turn depend on the absorbance. In the present study, we did the calculation of the chromatic coordinates, using the absorbance described as the sum of absorption lines. We applied the decomposition process described in previous reports $[10,15]$ to determine the parameters of these lines from the spectrum of absorbance shown in Figure 1.

Figure 1 shows the optical absorption spectrum of a Brazilian blue topaz of an irradiated sample submitted to heat treatment at $200^{\circ} \mathrm{C}$ for 24 hours. This spectrum shows a single maximum of transmittance at around $470 \mathrm{~nm}$. 


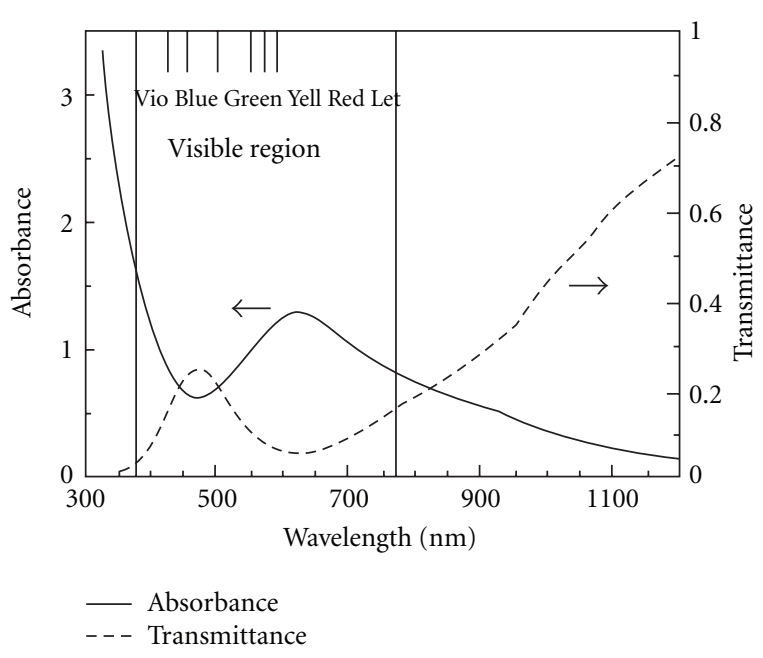

FIgURE 1: Optical absorption spectrum of blue topaz.

The maximum transmittance occurs at the intersection of the absorption band at $620 \mathrm{~nm}$ and the tail of an absorption in the ultraviolet region. Thus, for a good representation of the absorbance in the ultraviolet region, we analyzed the absorption spectra of thin sample of topaz. Figure 2 depicts the absorption spectra of a thin sample of irradiated topaz submitted to heat treatment at $200^{\circ} \mathrm{C}$ and $600^{\circ} \mathrm{C}$ and the results of the decomposition of these spectra as sum of Gaussian functions.

Figure 2(a) depicts as dots, the three lines obtained from the decomposition of the spectrum of irradiated topaz heated to $600^{\circ} \mathrm{C}$. The parameters of this spectrum decomposition are shown in Table 1. Although the line $60900 \mathrm{~cm}^{-1}$ is outside the observation range of our spectrometer and its location can not be determined with great accuracy, it indicates the presence of a strong absorption in the ultraviolet region.

The decomposition of the spectrum of topaz shows three lines at 60900,41630 , and $38510 \mathrm{~cm}^{-1}$. These lines are resistant to temperatures of $600^{\circ} \mathrm{C}$ and are similar to those three lines observed in spodumene heated to $400^{\circ} \mathrm{C}$ [15]. Similar lines were also observed in beryl [10]. In the absence of more experimental evidence to support a more consistent assignment, we will consider the assignments suggested in these works. Lines at around $61000 \mathrm{~cm}^{-1}$ were observed in oxygen-deficient silicon [29, 30], spodumene [15], and beryl. They were assigned using a first principle quantum-chemical study to a single diamagnetic oxygen vacancy ( $\equiv \mathrm{Si}-\mathrm{Si} \equiv)$. Therefore, we attribute the line at $60900 \mathrm{~cm}^{-1}$ determined in this work to a single diamagnetic oxygen vacancy ( $\equiv \mathrm{Si}-\mathrm{Si} \equiv$ ) $[31,32]$.

It was shown that in spodumene the lines at 40900 and $37400 \mathrm{~cm}^{-1}$ and in beryl the lines at 41400 and $36400 \mathrm{~cm}$ belong to a single defect. Two possible options were considered for assignments from quantum mechanical calculations TD-DFT for exchange correlation functional $\left(\mathrm{LDA} / 6-31+\mathrm{G}^{*}\right),\left(\mathrm{BLYP} / 6-31+\mathrm{G}^{*}\right)$, and (PW91/6+G*): a peroxy bridge $\left(\equiv \mathrm{Si}-\mathrm{O}-\mathrm{O}-\mathrm{Si} \equiv\right.$ ) with transitions $S_{0} \rightarrow S_{2}$ $37600-37840 \mathrm{~cm}^{-1}$ and $38900-41390 \mathrm{~cm}^{-1}$ and a silicon dangling bond defect $\left(=\mathrm{Si}\right.$ ) with transitions $S_{0} \rightarrow S_{2}$

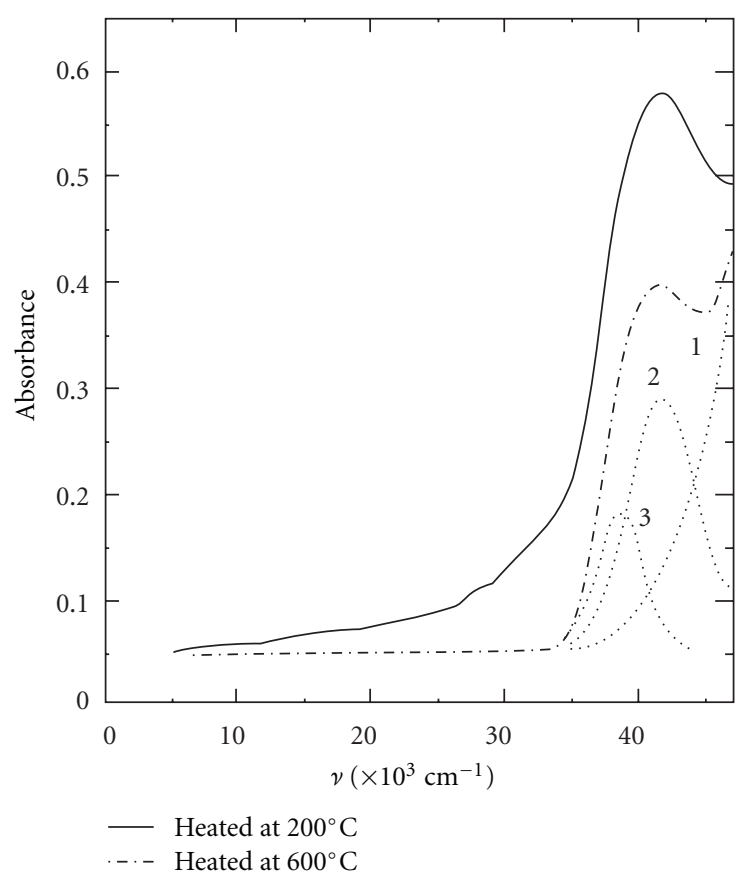

(a)

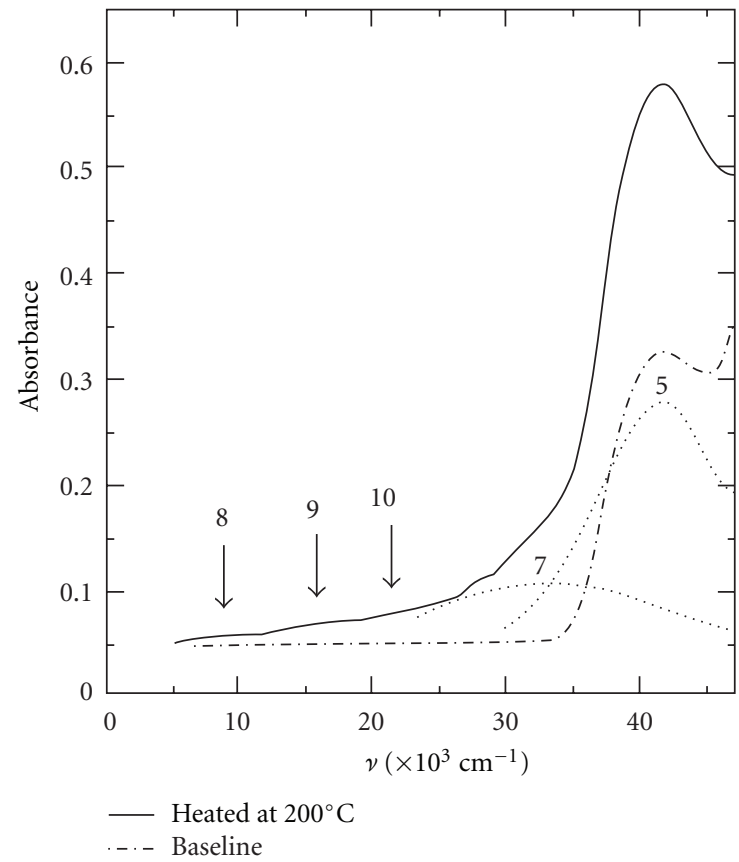

(b)

FIGURE 2: Optical absorption of irradiated thin sample of topaz heated at $200^{\circ} \mathrm{C}$ and $600^{\circ} \mathrm{C}$.

at $38300-38490 \mathrm{~cm}^{-1}$ and $41640-41960 \mathrm{~cm}^{-1}$ [32]. On the other hand, (B3LYP/6-31+ $\left.\mathrm{G}^{*}\right)$ has higher energy transition at 41070 and $48980 \mathrm{~cm}^{-1}$ for the peroxy bridge defect and 42260 and $47120 \mathrm{~cm}^{-1}$ for the silicon dangling bond defect, showing that the transition energies depend strongly on the exchange. To reduce the strong dependence of the exchange in the comparisons, we analyzed the differences in 
TABLE 1: Best-fitted parameters of the optical absorption spectra of thin sample of topaz heated at $600^{\circ} \mathrm{C}$ for Gaussian line shapes. The Gaussian amplitudes are given in absorbance units.

\begin{tabular}{lccc}
\hline Peak & $v_{0 i}\left(10^{3} \mathrm{~cm}^{-1}\right)$ & $\Delta v_{i}\left(10^{3} \mathrm{~cm}^{-1}\right)$ & Amplitude \\
\hline 1 & $60.9 \pm 2.0$ & $8.67 \pm 0.59$ & $5.46 \pm 0.89$ \\
2 & $41.63 \pm 0.40$ & $3.01 \pm 0.18$ & $0.648 \pm 0.025$ \\
3 & $38.51 \pm 0.12$ & $1.96 \pm 0.17$ & $0.297 \pm 0.034$ \\
\hline
\end{tabular}

transition energies between the two lines $S_{0} \rightarrow S_{2}$. In this case, the differences for $\left(\mathrm{LDA} / 6-31+\mathrm{G}^{*}\right),\left(\mathrm{BLYP} / 6-31+\mathrm{G}^{*}\right)$, (PW91/6-31+G*), and (B3LYP/6-31+G*) are 3790, 1450, 1210 , and $6460 \mathrm{~cm}^{-1}$ for the peroxy bridge defect and 3310 , 3470,3390 , and $6860 \mathrm{~cm}^{-1}$ for the silicon dangling bond defect, respectively. Since the difference between lines 2 and 3 from Table 1 is $3210 \mathrm{~cm}^{-1}$, we assign these lines to the silicon dangling bond defect (=Si:).

We note that in our samples the irradiation produces an intense brown color. Heating at $200^{\circ} \mathrm{C}$ eliminates this brown color. In some samples, a blue color remains and the absorption spectrum corresponds to that of Figure 1. In other samples the resulting color is slightly grayish or weakly bluish and corresponds to the absorption spectra of irradiated thin topaz heated at $200^{\circ} \mathrm{C}$. The thin sample after heat treatment at $600^{\circ} \mathrm{C}$ becomes transparent.

In spodumene, the spectrum of the sample heated at $400^{\circ} \mathrm{C}$ was not produced by irradiation. The spectrum of topaz heated at $600^{\circ} \mathrm{C}$ is similar to the spectrum of spodumene heated at $400^{\circ} \mathrm{C}$. Then, we assume that in topaz this spectrum is due to intrinsic defects not produced by irradiation. The irradiation causes the appearance of new defects that cause the appearance of new absorption lines. That is, the lines originating from the irradiation overlap the lines originating from intrinsic defects. For this reason, in the decomposition process of Figure 2(b) we subtract the spectrum band of topaz heated at $600^{\circ} \mathrm{C}$.

We began the process of decomposition of the spectra of topaz submitted to heat treatment at $200^{\circ} \mathrm{C}$ using as reference for the far ultraviolet region the lines observed in spodumene. The lines in irradiated spodumene at 33770 and $43160 \mathrm{~cm}^{-1}$ follow the same growth kinetics and were assigned to a single defect such as silanone $(=\mathrm{Si}=\mathrm{O})$. Calculations for silanone by TD-DFT with exchange correlation functional (B3LYP/6-31+G*) give transitions comparable with these lines [32]. A line at $16000 \mathrm{~cm}^{-1}$ in blue topaz was attributed by Krambrock et al. [24], considering the phenomenological theory of small bound polarons, to the fact that an $\mathrm{O}^{-}$hole center nearest to an acceptor defect is stabilized by lattice distortions.

We started the process of decomposition from the abovementioned three lines at 16000,33770 , and $43160 \mathrm{~cm}^{-1}$, using as the baseline the spectrum of topaz heated at $600^{\circ} \mathrm{C}$. Although the fit was satisfactory for the spectrum of Figure 2(b), we introduce two more lines to fit two small bumps on the main spectrum. These bumps at 8990 and $31470 \mathrm{~cm}^{-1}$ are sharp lines and are around the characteristic lines of $\mathrm{Fe}^{2+}$ in some distorted sites, as in beryl [5]. So, we could not determine the errors of these lines.
TABLE 2: Best-fitted parameters of the optical absorption spectra of thin sample of topaz heated at $200^{\circ} \mathrm{C}$ for Gaussian line shapes. The Gaussian amplitudes are given in absorbance units.

\begin{tabular}{lccc}
\hline Peak & $v_{0 i}\left(10^{3} \mathrm{~cm}^{-1}\right)$ & $\Delta \nu_{i}\left(10^{3} \mathrm{~cm}^{-1}\right)$ & Amplitude \\
\hline 4 & $41.77 \pm 0.27$ & $6.04 \pm 0.21$ & $0.426 \pm 0.030$ \\
5 & $32.0 \pm 1.3$ & $9.1 \pm 1.2$ & $0.103 \pm 0.014$ \\
6 & $31.47 \pm ?$ & $1.73 \pm ?$ & $0.026 \pm ?$ \\
7 & $16.3 \pm 2.0$ & $9.1 \pm 1.2$ & $0.029 \pm 0.006$ \\
8 & $8.99 \pm ?$ & $1.01 \pm ?$ & $0.004 \pm ?$ \\
\hline
\end{tabular}

The amplitude values for which the errors are indicated by "?" are very small and therefore they could not be evaluated. These lines were maintained to give stability to the optimization algorithm used in the determination of the parameters of the lines.

TABLE 3: Best-fitted parameters of the optical absorption spectra of thick sample (shown in Figure 1) of topaz heated at $200^{\circ} \mathrm{C}$ for Gaussian line shapes. The Gaussian amplitudes are given in absorbance units.

\begin{tabular}{lccc}
\hline Peak & $\nu_{0 i}\left(10^{3} \mathrm{~cm}^{-1}\right)$ & $\Delta \nu_{i}\left(10^{3} \mathrm{~cm}^{-1}\right)$ & Amplitude \\
\hline 5 & $33.04 \pm 0.19$ & $7.150 \pm 0.077$ & $2.760 \pm 0.047$ \\
7 & $15.986 \pm 0.064$ & $3.253 \pm 0.073$ & $1.204 \pm 0.031$ \\
9 & $10.92 \pm 0.20$ & $2.00 \pm 0.35$ & $0.297 \pm 0.019$ \\
\hline
\end{tabular}

Table 2 presents the values of the fitting parameters for the spectrum of irradiated thin topaz heated at $200^{\circ} \mathrm{C}$. The fitting process led to values of main lines at 32960 and $41770 \mathrm{~cm}^{-1}$, depicted as dots in Figure 2(b). The other three lines at 8990,16300 , and $31470 \mathrm{~cm}^{-1}$ are too small to be shown in Figure 2(b) and are indicated by arrows. The amplitude of the baseline was found to be $0.577 \pm 0.054$.

Table 3 presents the values of the fitting parameters for the thick sample of topaz. The decomposition with the baseline and two lines at about 16,000 and $33,000 \mathrm{~cm}^{-1}$ was obtained with acceptable accuracy. However, the long decay to lower energies suggests the presence of more lines in the infrared region. We introduced a line to simulate mathematically these unknown lines and provide stability to the process of decomposition. More rigorous experimental measurements need to be made so that such lines are properly identified. The amplitude of the baseline was found to be $0.746 \pm 0.016$.

\section{The Effect of Thickness on the Color}

Figure 1 shows that in the visible region there is a single minimum in the absorbance. It results from the combination of an absorption band in the region of $600 \mathrm{~nm}$ and an absorption tail from the ultraviolet region. This minimum happens when the derivative of the absorbance in relation to the wavelength is zero, that is, for $\lambda=\lambda_{m}$ we have $\alpha\left(\lambda_{m}\right)=\min$.

Here, $\left[P(\lambda) \bar{x}_{\lambda} \cdot \Delta \lambda\right],\left[P(\lambda) \bar{y}_{\lambda} \cdot \Delta \lambda\right]$, and $\left[P(\lambda) \bar{z}_{\lambda} \cdot \Delta \lambda\right]$ are scheduled for visual angle $2^{\circ}$, and the CIE illuminants are $\mathrm{A}$ and $\mathrm{D}_{65}$. The A illuminant represent a light source of a tungsten lamp light at $2857 \mathrm{~K}$ color temperature. The $\mathrm{D}_{65}$ illuminant represents a light source of average daylight and has a correlated color temperature of approximately $6500 \mathrm{~K}$. 
The CIE source coordinates are $\mathrm{A} \equiv(0.4476,0.4075)$ and $\mathrm{D}_{65} \equiv(0.31271,0.32902)$.

The colors calculated for blue topaz, with illuminants A and D65, and parameters of absorbance of Table 3, are shown in Figure 3. The thickness of the sample color changes along a color path. We see that the color path moves through the 1931 Chromaticity Diagram reaching its border in the limit of the maximum transmittance around $\lambda_{0}=470 \mathrm{~nm}$.

Purity and dominant wavelength for topaz, depending on sample thickness $\ell$, are shown in Figure 4 . This shows that the purity and dominant wavelength change continuously as functions of thickness through a pathway, from illuminant $(\ell=0)$ to borderline values $(\ell \rightarrow \infty)$. A thick sample shows a very low purity but a rapid increase in purity, reaching saturation at thicknesses greater than $5 \mathrm{~mm}$. On the other hand, the dominant wavelength of a thick sample decreases continuously from $493 \mathrm{~nm}$ to $470 \mathrm{~nm}$ for illuminant A and from $483 \mathrm{~nm}$ to $470 \mathrm{~nm}$ for illuminant $\mathrm{D}_{65}$.

As seen in Figure 4, under a certain illuminant, the "chromatic path" starts at the illuminant point (very thin) and finishes at the borderline (very thick). This observation can be demonstrated by theoretically analyzing the path in the Chromaticity Diagram written in continuous forms as follows:

$$
\begin{aligned}
& x=\frac{\int_{\lambda=380}^{770} P_{L}(\lambda) T(\lambda) \bar{x}_{\lambda} d \lambda}{\int_{\lambda=380}^{770} P_{L}(\lambda) T(\lambda)\left(\bar{x}_{\lambda}+\bar{y}_{\lambda}+\bar{z}_{\lambda}\right) d \lambda}, \\
& y=\frac{\int_{\lambda=380}^{770} P_{L}(\lambda) \cdot T(\lambda) \bar{y}_{\lambda} d \lambda}{\int_{\lambda=380}^{770} P_{L}(\lambda) T(\lambda)\left(\bar{x}_{\lambda}+\bar{y}_{\lambda}+\bar{z}_{\lambda}\right) d \lambda} .
\end{aligned}
$$

Equation (4) shows that the maximum of the transmittance corresponds to the minimum of the absorbance. Using the definition for the line width $T\left(\lambda_{1 / 2}\right)=T\left(\lambda_{m}\right) / 2$, we obtain the relationship:

$$
\ln 2=\left[\alpha\left(\lambda_{1 / 2}\right)-\alpha\left(\lambda_{m}\right)\right] \ell .
$$

Therefore, for very large values of $\ell$ the value of the line width tends to be very small.

In Figure 5, we show this property by overlapping the normalized transmittances for several thicknesses. The normalization is required since its absolute value decrease to zero when thickness becomes infinite [33-35]. The dots are the experimentally observed absorbances. The solid lines show the normalized transmittance dependence on $\lambda$. We see that transmittance becomes sharper as thickness increases.

In the limit of large thickness, the transmittance becomes very small and so both terms, numerator and denominator of (7) tend to zero. However, considering that in the limit of large values of thickness the line width of the transmittance becomes very sharp, we can approximate the integral to that

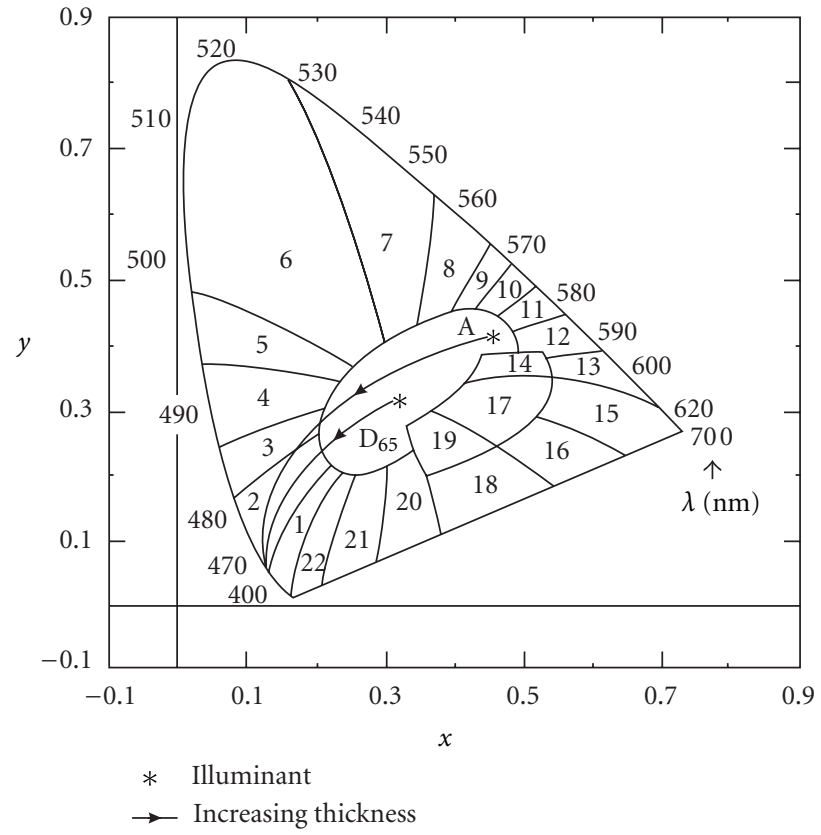

Figure 3: $19312^{\circ}$ CIE Chromaticity Diagram containing the A and $\mathrm{D}_{65}$ sources and topaz color line path depending on thickness. The regions represent the following colors: (1) purplish blue, (2) blue, (3) greenish blue, (4) blue-green, (5) bluish-green, (6) green, (7) yellowish-green, (8) yellow-green, (9) greenish-yellow, (10) yellow, (11) orange-yellow, (12) orange, (13) reddish-orange, (14) yellowish-pink, (15) red, (16) purplish-red, (17) pink, (18) reddishpurple, (19) purplish-pink, (20) reddish-purple, (21) purple, and (22) violet.

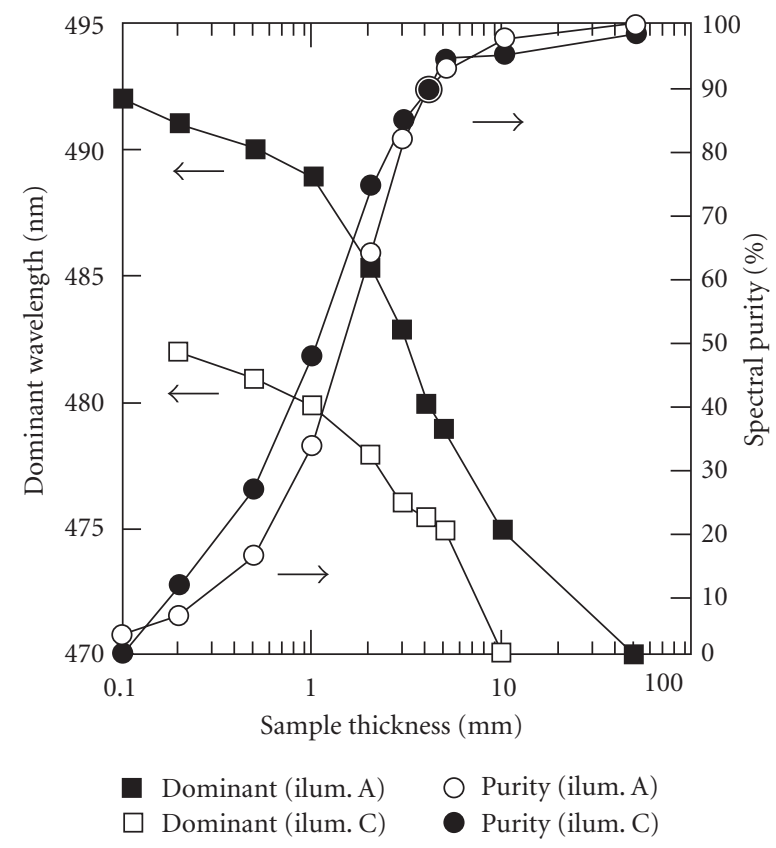

FIGURE 4: Dominant wavelength (in $\mathrm{nm}$ ) and spectral purity (in \%) as function of sample thickness (in $\mathrm{mm}$ ) for blue topaz. 


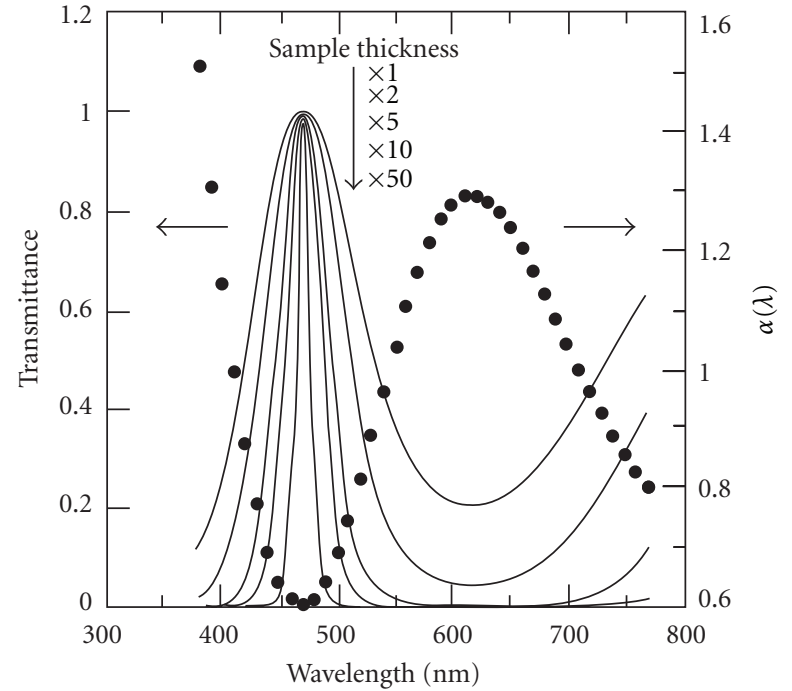

Figure 5: Dependence on thickness of normalized absorbance and transmittance of blue topaz.

value calculated at $\lambda=\lambda_{m}$. Then, using this property we rewrite (7) as

$$
\begin{aligned}
x & \rightarrow \frac{P_{L}\left(\lambda_{m}\right) T\left(\lambda_{m}\right) \bar{x}_{\lambda_{m}} \Delta \lambda}{P_{L}\left(\lambda_{m}\right) T\left(\lambda_{m}\right)\left(\bar{x}_{\lambda_{m}}+\bar{y}_{\lambda_{m}}+\bar{z}_{\lambda_{m}}\right) \Delta \lambda} \\
& =\frac{\bar{x}_{\lambda_{m}}}{\left(\bar{x}_{\lambda_{m}}+\bar{y}_{\lambda_{m}}+\bar{z}_{\lambda_{m}}\right)}, \\
y & \longrightarrow \frac{P_{L}\left(\lambda_{m}\right) T\left(\lambda_{m}\right) \bar{y}_{\lambda_{m}} \Delta \lambda}{P_{L}\left(\lambda_{m}\right) T\left(\lambda_{m}\right)\left(\bar{x}_{\lambda_{m}}+\bar{y}_{\lambda_{m}}+\bar{z}_{\lambda_{m}}\right) \Delta \lambda} \\
& =\frac{\bar{x}_{\theta, \lambda_{m}}}{\left(\bar{x}_{\lambda_{m}}+\bar{y}_{\lambda_{m}}+\bar{z}_{\lambda_{m}}\right)} .
\end{aligned}
$$

These coordinates are the coordinates of the borderline of the 1931 Chromaticity Diagram at the wavelength $\lambda=\lambda_{m}$.

As we mentioned above, the dominant wavelength $\left(x_{\lambda}\right.$, $\left.y_{\lambda}\right)$ is the point in the diagram borderline crossed by the straight line in the direction of the light source to the color coordinates. The dominant wavelength $\lambda_{d}$, corresponding to the $\left(x_{\lambda}, y_{\lambda}\right)$ coordinates in the limit of large thickness, coincides with the color coordinate at the wavelength $\lambda=\lambda_{m}$. Then, according to (5), the purity becomes $P_{L}=1$.

\section{Conclusion}

In the present work, we applied the analysis of the optical absorption spectral decomposition of irradiated topaz submitted to heat treatment at $200^{\circ} \mathrm{C}$ and $600^{\circ} \mathrm{C}$. The optical absorption spectrum of blue topaz was analyzed using several Gaussians. The decomposition of the topaz spectra submitted to heat treatment at $600^{\circ} \mathrm{C}$ revealed three lines. The parameters were obtained using a grid optimization method. Discussions, taking into account highly accurate quantum mechanical calculations $[31,32]$ and results in spodumene
[15] and beryl [10], led to assigning the lines at 41630 and $38510 \mathrm{~cm}^{-1}$ to a silicon dangling bond defect (=Si:) and the line at $60900 \mathrm{~cm}^{-1}$ to an oxygen vacancy defect ( $\equiv \mathrm{Si}-$ $\mathrm{Si} \equiv)$. The decomposition of the spectra of thin and thick samples submitted to heat treatment at $200^{\circ} \mathrm{C}$ shows a line at around $16000 \mathrm{~cm}^{-1}$ due to an $\mathrm{O}^{-}$hole center nearest to an acceptor defect being stabilized by lattice distortions [24]. Furthermore, two lines around 32000 and $42000 \mathrm{~cm}^{-1}$, based on similar lines observed in spodumene, suggested for assignment a single defect such as silanone $(=\mathrm{Si}=\mathrm{O})$.

The calculated transmittance showed a remarkable sharpening with increasing thickness. The CIE coordinates of color go through the pathway from the illuminant coordinates to the borderline of the Chromaticity Diagram, reaching them at coordinates corresponding to the maximum transmittance as the thickness increases. The color purity increases with increasing thickness, and the dominant wavelength reaches the wavelength of maximum transmittance.

\section{Acknowledgments}

This work was supported by Grants from FAPESP, FINEP, and CNPq.

\section{References}

[1] B. Henderson, Color, Contemporary Physics, vol. 19, p. 225, 1978.

[2] R. D. Lozano, El Color y Su Medición, Editorial Americalee, Buenos Aires, Argentina, 1978.

[3] G. Wyszecki and W. S. Stiles, Color Science: Concepts and Methods, Quantitative Data and Formulae, John Wiley \& Sons, New York, NY, USA, 1982.

[4] R. R. Viana, I. Mänttäri, H. Kunst, and H. Jordt-Evangelista, "Age of pegmatites from eastern Brazil and implications of mica intergrowths on cooling rates and age calculations," Journal of South American Earth Sciences, vol. 16, no. 6, pp. 493501, 2003.

[5] A. R. Blak, S. Isotani, and S. Watanabe, "Optical absorption and electron spin resonance in blue and green natural beryl," Physics and Chemistry of Minerals, vol. 8, no. 4, pp. 161-166, 1982.

[6] S. Isotani, W. W. Furtado, R. Antonini, and O. L. Dias, "Lineshape and thermal kinetics analysis of the $\mathrm{Fe}^{2+}$ band in Brazilian green beryl," American Mineralogist, vol. 74, no. 3-4, pp. 432-438, 1989.

[7] K. Krambrock, M. V. B. Pinheiro, K. J. Guedes et al., "Radiation-induced centers in Cs-rich beryl studied by magnetic resonance, infrared and optical spectroscopy," Nuclear Instruments and Methods in Physics Research B, vol. 191, no. 1-4, pp. 285-290, 2002.

[8] R. R. Viana, H. Jordt-Evangelista, G. M. da Costa, and W. B. Stern, "Characterization of beryl (aquamarine variety) from pegmatites of Minas Gerais, Brazil," Physics and Chemistry of Minerals, vol. 29, no. 10, pp. 668-679, 2002.

[9] J. C. R. Mittani, S. Watanabe, J. F. D. Chubaci, M. Matsuoka, D. L. Baptista, and F. C. Zawislak, " $\gamma$-radiation effects on colourless silicates of beryl," Nuclear Instruments and Methods in Physics Research B, vol. 191, no. 1-4, pp. 281-284, 2002. 
[10] S. Isotani, A. R. Blak, and S. Watanabe, "UV optical absorption spectra analysis of beryl crystals from Brazil," Physica B, vol. 405, no. 6, pp. 1501-1508, 2010.

[11] C. T. Dotto and S. Isotani, "Irradiation and heating effects in amethyst crystals from brazil ," Radiation Effects and Defects in Solids, vol. 117, no. 4, pp. 355-361, 1991.

[12] A. T. Fujii and S. Isotani, "Optical absorption study of five varieties of spodumene," Anais da Academia Brasileira de Ciências, vol. 60 , no. 2 , pp. $127-135,1988$.

[13] A. S. Ito and S. Isotani, "Heating effects on the optical absorption spectra of irradiated, natural spodumene," Radiation Effects and Defects in Solids, vol. 116, no. 4, pp. 307-314, 1991.

[14] S. Isotani, A. T. Fujii, R. Antonini, W. M. Pontuschka, S. R. Rabbani, and W. W. Furtado, "Optical absorption study of radiation and thermal effects in Brazilian samples of spodumene," Anais da Academia Brasileira de Ciências, vol. 63, no. 2, pp. 127-139, 1991.

[15] S. Isotani, K. Watari, A. Mizukami, W. Bonventi Jr., and A. S. Ito, "UV optical absorption spectra analysis of spodumene crystals from Brazil," Physica B, vol. 391, no. 2, pp. 322-330, 2007.

[16] S. C. Lind and L. Bardwell, "The coloring and thermophosphorescence produced in transparent minerals and gems by radium radiation," American Mineralogist, vol. 8, pp. 171-180, 1923.

[17] K. Nassau and B. E. Prescott, "Blue and brown topaz produced by gamma irradiation," American Mineralogist, vol. 60, pp. 705-709, 1975.

[18] A. R. P. L. Albuquerque, S. Isotani, and S. P. Morato, "Irradiation and heating effects in topaz crystals from minas cerais, Brazil," Radiation Effects and Defects in Solids, vol. 106, no. 12, pp. 143-150, 1988.

[19] V. Priest, D. L. Cowan, D. G. Reichel, and F. K. Ross, "A dangling-silicon-bond defect in topaz," Journal of Applied Physics, vol. 68, no. 6, pp. 3035-3037, 1990.

[20] D. N. da Silva, K. J. Guedes, M. V. B. Pinheiro, S. Schweizer, J. M. Spaeth, and K. Krambrock, "The O-(Al2) centre in topaz and its relation to the blue colour," Physica Status Solidi C, vol. 2, no. 1, pp. 397-400, 2005.

[21] D. N. da Silva, K. J. Guedes, M. V. B. Pinheiro, J. M. Spaeth, and K. Krambrock, "The microscopic structure of the oxygenaluminium hole center in natural and neutron irradiated blue topaz," Physics and Chemistry of Minerals, vol. 32, no. 5-6, pp. 436-441, 2005.

[22] A. S. Leal, K. Krambrock, L. G. M. Ribeiro, M. A. B. C. Menezes, P. Vermaercke, and L. Sneyers, "Study of neutron irradiation-induced colors in Brazilian topaz," Nuclear Instruments and Methods in Physics Research A, vol. 580, no. 1, pp. 423-426, 2007.

[23] O. F. Schirmer, "O $\mathrm{O}^{-}$bound small polarons in oxide materials," Journal of Physics: Condensed Matter, vol. 18, no. 43, article R667, 2006.

[24] K. Krambrock, L. G. M. Ribeiro, M. V. B. Pinheiro, A. S. Leal, M. A. B. C. Menezes, and J. M. Spaeth, "Color centers in topaz: comparison between neutron and gamma irradiation," Physics and Chemistry of Minerals, vol. 34, no. 7, pp. 437-444, 2007.

[25] M. B. de Camargo and S. Isotani, "Optical absorption spectroscopy of natural and irradiated pink tourmaline," American Mineralogist, vol. 73, pp. 172-180, 1988.

[26] S. Isotani and A. T. Fujii, "A grid procedure applied to the determination of parameters of a kinetic process," Computer Physics Communications, vol. 151, no. 1, pp. 1-7, 2003.

[27] O. L. Dias, A. R. P. L. Albuquerque, and S. Isotani, "X-Ray fluorescence analysis of Fe, Mn, Cr and V in natural Silicate
Crystals," Anais da Academia Brasileira de Ciências, vol. 55, p. $173,1983$.

[28] J. J. Markham, "Interaction of normal modes with electron traps," Reviews of Modern Physics, vol. 31, no. 4, pp. 956-989, 1989.

[29] H. Hosono, Y. Abe, H. Imagawa, H. Imai, and K. Arai, "Experimental evidence for the Si-Si bond model of the 7.6-eV band in $\mathrm{SiO}_{2}$ glass," Physical Review B, vol. 44, no. 21, pp. 1204312045, 1991.

[30] H. Nishikawa, R. Nakamura, Y. Ohki, and Y. Hama, "Correlation of preexisting diamagnetic defect centers with induced paramagnetic defect centers by ultraviolet or vacuum-ultraviolet photons in high-purity silica glasses," Physical Review B, vol. 48, no. 21, pp. 15584-15594, 1993.

[31] G. Pacchioni and G. Ieranò, "On the origin of the 5.0 and $7.6 \mathrm{eV}$ absorption bands in oxygen deficient $\alpha$-quartz and amorphous silica. A first principles quantum-chemical study," Journal of Non-Crystalline Solids, vol. 216, pp. 1-9, 1997.

[32] K. Raghavachari, D. Ricci, and G. Pacchioni, "Optical properties of point defects in $\mathrm{SiO}_{2}$ from time-dependent density functional theory," Journal of Chemical Physics, vol. 116, no. 2, pp. 825-831, 2002.

[33] R. G. Burns, Mineralogical Applications of Crystal Field Theory, Cambridge University Press, Cambridge, UK, 1970.

[34] R. Kuehni, Colour: An Introduction to Practice and Principle, John Wiley \& Sons, New York, NY, USA, 1997.

[35] Y. Liu, J. Shigley, and A. Halvorsen, "Colour hue change of a gem tourmaline from Umba Valley, Tanzania," Journal of Gemmology, vol. 26, no. 6, pp. 386-396, 1999. 

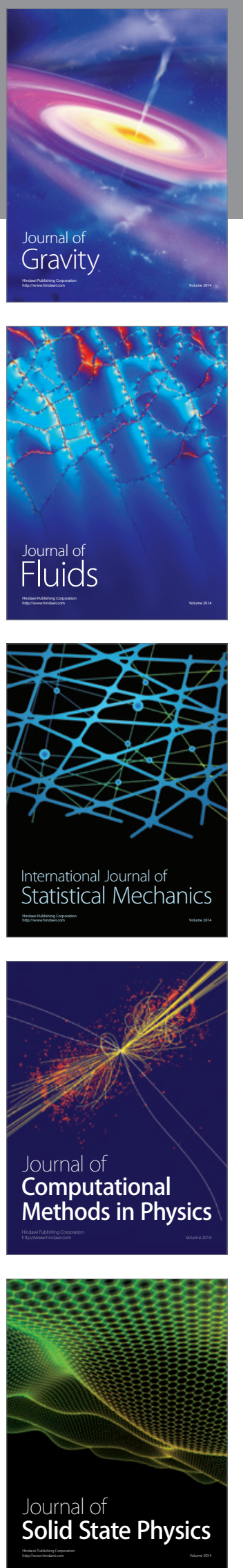

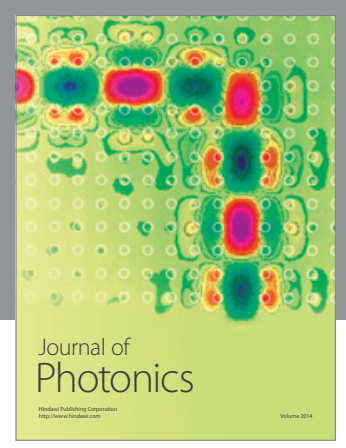

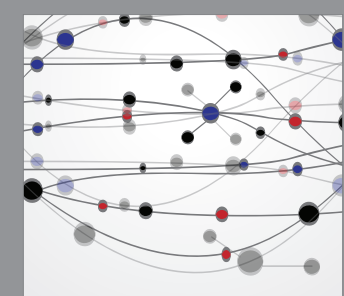

The Scientific World Journal
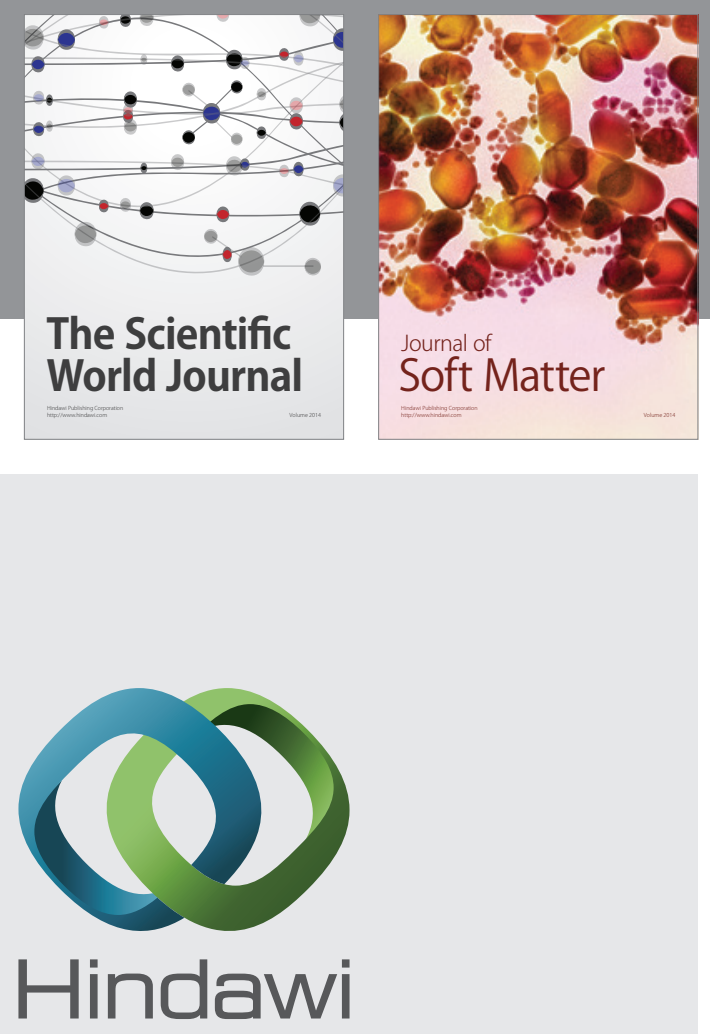

Submit your manuscripts at

http://www.hindawi.com
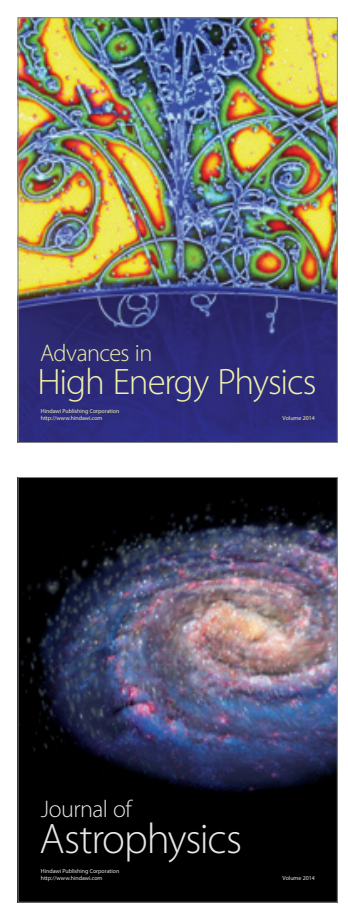
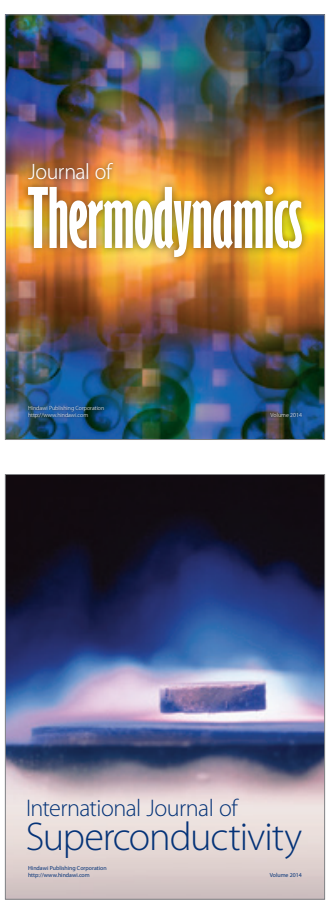
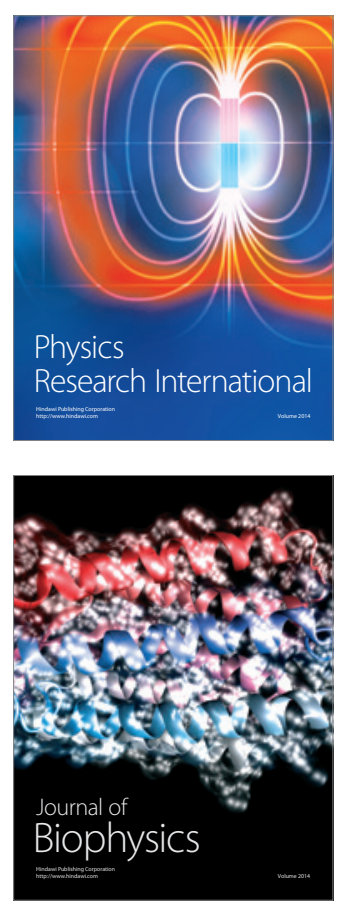
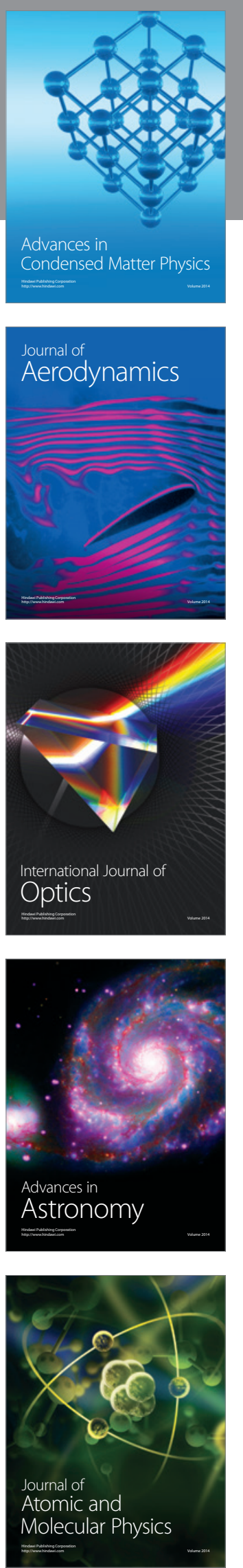